\title{
Foxo3 is required for the regulation of oxidative stress in erythropoiesis
}

\author{
Dragan Marinkovic, ${ }^{1}$ Xin Zhang, ${ }^{1}$ Safak Yalcin, ${ }^{1}$ Julia P. Luciano, ${ }^{1}$ \\ Carlo Brugnara, ${ }^{2}$ Tara Huber, ${ }^{1}$ and Saghi Ghaffari $1,3,4,5$
}

1Department of Gene and Cell Medicine, Mount Sinai School of Medicine, New York, New York, USA. 2Department of Laboratory Medicine, Children's Hospital, Harvard Medical School, Boston, Massachusetts, USA. ${ }^{3}$ Black Family Stem Cell Institute, ${ }^{4}$ Department of Molecular, Cell, and Developmental Biology, and ${ }^{5}$ Department of Medicine, Division of Hematology and Medical Oncology, Mount Sinai School of Medicine, New York, New York, USA.

\begin{abstract}
Erythroid cells accumulate hemoglobin as they mature and as a result are highly prone to oxidative damage. However, mechanisms of transcriptional control of antioxidant defense in erythroid cells have thus far been poorly characterized. We observed that animals deficient in the forkhead box $\mathrm{O} 3$ (Foxo3) transcription factor died rapidly when exposed to erythroid oxidative stress-induced conditions, while wild-type mice showed no decreased viability. In view of this striking finding, we investigated the potential role of Foxo3 in the regulation of ROS in erythropoiesis. Foxo3 expression, nuclear localization, and transcriptional activity were all enhanced during normal erythroid cell maturation. Foxo3-deficient erythrocytes exhibited decreased expression of ROS scavenging enzymes and had a ROS-mediated shortened lifespan and evidence of oxidative damage. Furthermore, loss of Foxo3 induced mitotic arrest in erythroid precursor cells, leading to a significant decrease in the rate of in vivo erythroid maturation. We identified ROS-mediated upregulation of p21 $1^{C I P 1 / W A F 1 / S d i 1}$ (also known as Cdkn1a) as a major contributor to the interference with cell cycle progression in Foxo3-deficient erythroid precursor cells. These findings establish an essential nonredundant function for Foxo3 in the regulation of oxidative stress, cell cycle, maturation, and lifespan of erythroid cells. These results may have an impact on the understanding of human disorders in which ROS play a role.
\end{abstract}

\section{Introduction}

The maintenance of highly regulated mechanisms to control ROS levels is essential for normal cellular homeostasis. ROS such as superoxide anions and hydrogen peroxide are byproducts of oxidative metabolism and are involved in many signaling processes (1). However, excess accumulation of ROS can result from defects in ROS scavenging and is believed to have an impact on cellular aging and the senescence process. Conversely, the ability to withstand oxidative stress has been correlated with enhanced longevity in several species (1). Abnormal accumulation of ROS has been implicated in the pathogenesis of various diseases such as ataxia telangiectasia and Fanconi anemia $(2,3)$.

The forkhead homolog DAF-16, the activation of which results in a significant increase in the lifespan of Caenorhabditis elegans, is a major mediator of defense against oxidative stress $(4,5)$. Forkhead box O1 (FOXO1), FOXO3a, FOXO4, and FOXO6 belong to the forkhead family of winged helix transcription factors and are the human homologs of DAF-16 (reviewed in ref. 6). Similar to DAF-16, activation of FOXO3a in cultured fibroblasts and neuronal cells results in resistance to oxidative stress $(7,8)$. This FOXO3a effect is mediated via upregulated transcription of the ROS scavenging enzymes superoxide dismutase 2 (SOD2, also known as MnSOD) and catalase $(7,8)$. FOXO3a responds to cellular stress (including to oxidative stress) by inducing cell cycle arrest, repair of damaged DNA, and apoptosis via upregulation of genes that control

Nonstandard abbreviations used: CDK, cyclin-dependent kinase; EKLF, erythroid Kruppel-like factor; Epo, erythropoietin; EpoR, erythropoietin receptor; Foxo3, forkhead box O3; NAC, N-acetyl-L-cysteine; QRT-PCR, quantitative real-time RT-PCR; SOD2, superoxide dismutase 2 .

Conflict of interest: The authors have declared that no conflict of interest exists. Citation for this article: J. Clin. Invest. 117:2133-2144 (2007). doi:10.1172/JCI31807. these processes (9-13). These functions are similar to those associated with the p53 tumor suppressor protein, with which FOXO3a shares several downstream anti-stress targets (14).

Female mice deficient in Foxo3 (murine homolog of human FOXO3a) exhibit global follicular activation that results in early ovarian follicle depletion and leads to a syndrome reminiscent of premature ovarian failure $(15,16)$. In addition, gene-trap mutant mice have been reported to exhibit inflammatory lymphoid hyperplasia (17) (Expression of a gene-trap cassette from the endogenous promoter results in a fusion transcript that encodes for a truncated and nonfunctional version of the cellular protein and the reporter/selectable marker.). It is unknown whether Foxo3 (or FOXO3a) contributes to the physiological control of oxidative stress in vivo.

FoxO proteins are downstream targets of the highly conserved PI3K/AKT signaling pathway (6). In response to receptor binding to factors such as insulin and erythropoietin $($ Epo) $(18,19)$ or oncogenic stimuli such as the breakpoint cluster region-v-Abl Abelson murine leukemia viral oncogene homolog (BCR-ABL) oncoprotein $(13,20)$, the AKT serine threonine kinase phosphorylates nuclear FoxO on 3 conserved residues. Phosphorylated FoxO binds to 14-3-3 adaptor proteins and translocates to the cytosol where, away from their transcriptional targets, FoxO is unable to induce gene expression (12). Conversely, stress stimuli or inhibition of PI3K/AKT by cytokine withdrawal results in nuclear localization and activation of FoxO (6).

Regulation of oxidative stress is particularly important to erythropoiesis and the production of mature erythrocytes that results from proliferation, survival, and differentiation of erythroid cell progenitors following the binding of Epo to its receptor (21). Erythroid precursors synthesize and accumulate hemoglobin as they mature. Circulating erythrocytes carry oxygen bound to hemoglobin and as such are highly prone to oxidative damage (22). Consequently, erythrocytes are exposed to some of the highest levels of 
Table 1

Erythrocyte parameters

\begin{tabular}{lrcc} 
Parameters & \multicolumn{1}{c}{ WT } & \multicolumn{1}{c}{ Foxo3 } & $\boldsymbol{P}$ \\
$\mathrm{rbc}\left(\times 10^{6} / \mu \mathrm{l}\right)$ & $9.89 \pm 0.49$ & $8.87 \pm 0.16$ & 0.04 \\
$\mathrm{Hgb}(\mathrm{g} / \mathrm{dl})$ & $14.88 \pm 0.79$ & $14.32 \pm 0.76$ & 0.31 \\
$\mathrm{Hct}(\%)$ & $45.52 \pm 2.20$ & $46.69 \pm 0.86$ & 0.32 \\
$\mathrm{MCH}(\mathrm{pg})$ & $14.25 \pm 0.10$ & $15.82 \pm 0.17$ & $1.03 \times 10^{-8}$ \\
$\mathrm{MCHC}(\mathrm{g} / \mathrm{dl})$ & $30.21 \pm 0.50$ & $30.61 \pm 0.39$ & 0.27 \\
$\mathrm{MCV}(\mathrm{fL})$ & $47.34 \pm 0.73$ & $52.00 \pm 0.72$ & $8.59 \times 10^{-5}$ \\
$\mathrm{RDW}(\%)$ & $14.45 \pm 0.05$ & $16.19 \pm 0.19$ & $3.71 \times 10^{-6}$
\end{tabular}

Results for WT $(n=10)$ and Foxo3-- $(n=21)$ are shown as mean \pm SEM. $\mathrm{Hgb}$, hemoglobin; Hct, hematocrit; $\mathrm{MCH}$, mean corpuscular hemoglobin; $\mathrm{MCHC}, \mathrm{MCH}$ concentration; MCV, mean corpuscular volume; RDW, red cell distribution width.

oxidative stress conditions in the body. Consistent with this, as seen in genetic deficiencies of enzymes involving pathways that maintain intracellular reductive molecules or of molecules that protect globin, compromised protection from ROS results in diseases of red blood cells that involve a shortened lifespan of these cells and in hemolysis, which leads to anemia (22-25). Not surprisingly, erythroid cells in healthy individuals contain a strong arsenal of antioxidant enzymes that protect the cells against oxygen radicals (26). Importantly, accumulation of ROS has a significant impact on the senescence of circulating erythrocytes and their limited lifespan (27).

Despite the importance of the regulation of oxidative stress in normal and altered erythropoiesis, transcriptional programs that regulate the expression of antioxidants in erythroid cells are unknown. Since mature erythrocytes are enucleated, important transcriptional regulation must take place prior to enucleation and release of erythrocytes to the peripheral circulation.

We have recently shown an important role for the PI3K/AKT signaling pathway, downstream of the Epo receptor (EpoR), in supporting primary erythroid progenitor cell differentiation and maturation $(28,29)$. Here we examine in detail the physiological function of Foxo3 in the regulation of erythropoiesis in wild-type and Foxo3-deficient mice. We found that expression, nuclear localization, and transcriptional activity of Foxo3 were enhanced during in vivo maturation of erythroid precursor cells. In addition, we show that loss of Foxo3 reduced the lifespan of circulating erythrocytes and induced a mitotic arrest in erythroid precursor cells, resulting in a significant decrease in the rate of erythroid cell maturation. These effects were mediated by ROS, as demonstrated by rescue of Foxo3deficient phenotypes using ROS scavengers. Finally, we show that loss of Foxo3 led to rapid death of the animal in response to an oxidative stress-induced enhanced hemolysis together with a defective erythroid cell production. We analyze the molecular mechanisms of this defective response. Our findings establish a pivotal, nonredundant role for Foxo3 in the regulation of oxidative stress, cell cycle, maturation, and lifespan of erythroid cells during erythropoiesis.

\section{Results}

Foxo3 is required for antioxidant response in vivo. At the steady state, despite a slight decrease in the numbers of circulating erythrocytes, Foxo3-deficient mice did not exhibit any anemia, and their hemoglobin and hematocrit were normal (Table 1).

To investigate whether Foxo3 plays any role in the in vivo regulation of oxidative stress in mammals, Foxo3-deficient mice were challenged with phenylhydrazine. Phenylhydrazine treatment (30) induces oxidative denaturation of hemoglobin, resulting in rapid destruction and significant loss of erythrocytes (hemolysis) and leading to anemia characterized by a decrease in hemoglobin concentration and hematocrit levels. In response to phenylhydrazine-induced hemolytic anemia, bone marrow and spleen erythropoiesis are stimulated releasing prematurely erythrocyte precursors, namely reticulocytes, into the bloodstream. Thus, reticulocyte content of blood reflects the severity of the hemolytic anemia and the degree of compensatory response of bone marrow and spleen to phenylhydrazine.

As anticipated, wild-type mice subjected to $100 \mathrm{mg} / \mathrm{kg}$ of phenylhydrazine recovered within 10 days from hemolytic anemia (Figure 1), during which red blood cell numbers and hematocrit and reticulocyte levels were corrected (data not shown) and animals survived the treatment (Figure 1). However, Foxo3 $3^{-/-}$ mice died rapidly with severe anemia within 72 hours (Figure 1 and data not shown) after injection of phenylhydrazine, suggesting that $\mathrm{Foxo3}^{-/-}$mice are hypersensitive to phenylhydrazine-induced oxidative stress. It is noteworthy that Foxo3-deficient mice induced the expected rapid reticulocyte response to lower doses of phenylhydrazine $(50 \mathrm{mg} / \mathrm{kg})$, as did wild-type mice. However, the level of reticulocytes remained high in Foxo3-deficient mice, about 4-fold that of wild-type mice (data not shown). These results suggested that the erythropoietic response at the progenitor level in Foxo3-deficient mice is not altered. Together these results indicate that Foxo3 may be required for the antioxidative stress response in vivo.

We hypothesized that Foxo3-deficient mice may suffer from a steady-state oxidative stress condition. To this end, the concentration of ROS in Foxo3 mutant erythrocytes was measured at the basal level (Figure 1, B and C). Using 5-(and 6-)-chloromethyl-2',7'dichlorodihydrofluorescein diacetate, a peroxide-sensitive probe, ROS were found to be significantly more concentrated in $\mathrm{FoxO}_{0} 3^{-/-}$ than in wild-type erythrocytes (Figure 1, B and C), in the absence of any exogenously added peroxide.

Protein oxidation results in introduction of carbonyl moieties to amino acid side chains (31). In agreement with high ROS concentration, several proteins were highly oxidized in Foxo3-deficient erythroid cells, as measured by western blot analysis of carbonyl groups reactive with 2,4-dinitrophenylhydrazine (2- to 3- fold the level observed in wild-type cells; Figure 1, D and E). Despite erythroid protein oxidation, Foxo3-deficient mice did not exhibit any anemia probably due to an expansion of extramedullary erythropoietic tissue (see Supplemental Figure 1A; supplemental material available online with this article; doi:10.1172/JCI31807DS1).

To further evaluate the potential impact of ROS, erythrocyte lifespan was measured in vivo. Animals were injected with $N$-hydroxysulfosuccinimide-biotin) (32) and analyzed for survived biotin-labeled erythrocytes every 5 days. These experiments showed that Foxo3-deficient erythrocyte lifespan was significantly shortened (Figure 2A), associated with an increased width of erythrocyte volume distribution that is often seen in hemolysis (Table 1). In addition, Foxo $3^{-/-}$reticulocyte index was 4-fold higher than that of wild-type mice (Figure $2 \mathrm{~B}$ ), reflecting the degree of compensatory response of Foxo $3^{-/-}$hematopoietic organs to hemolysis. Based on these results, we concluded that $\mathrm{FoxO}^{-/-}$mice exhibit a chronic hemolysis in the absence of anemia.

In agreement with a role for ROS in reducing erythrocyte lifespan, the peritoneal injection of $\mathrm{N}$-acetyl-L-cysteine (NAC), a generic ROS scavenger, significantly improved the lifespan of $\mathrm{Foxo3}^{-/-}$erythrocytes (Figure 3A). Consistent with these results, NAC treatment also 


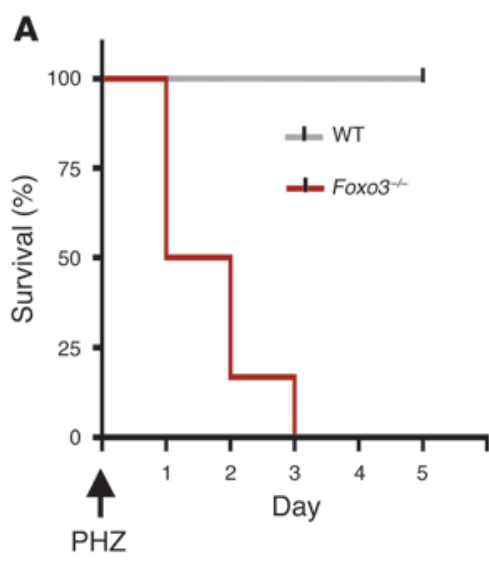

D

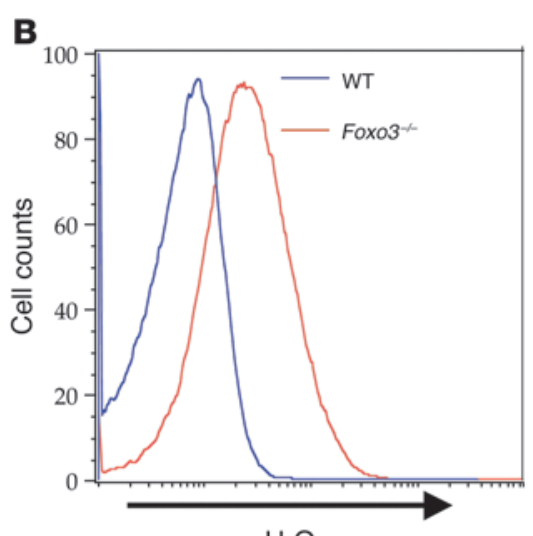

$\mathrm{H}_{2} \mathrm{O}_{2}$

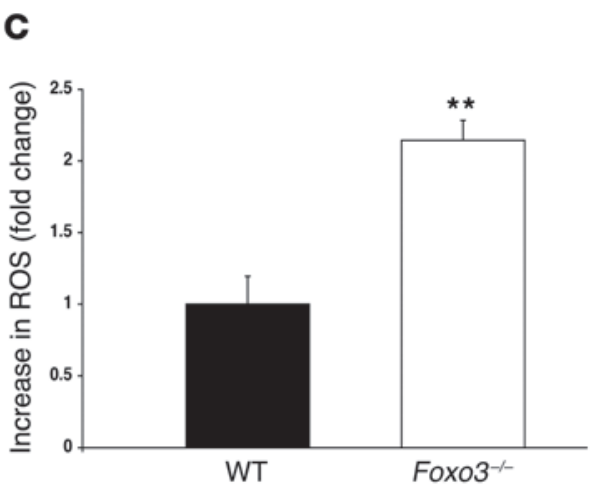

E
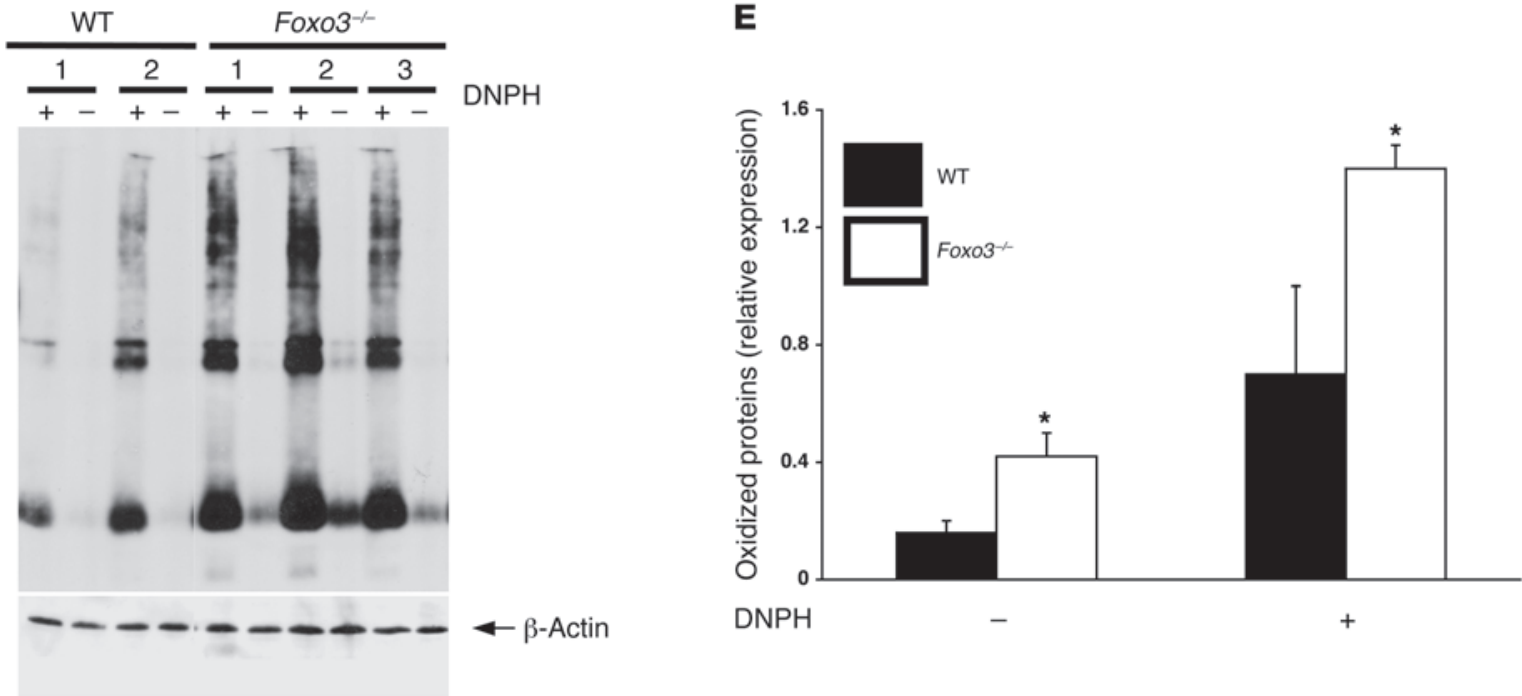

\section{Figure 1}

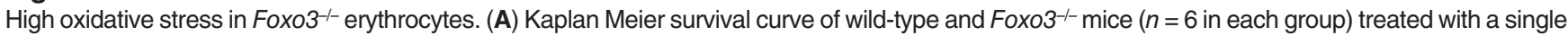
dose of intraperitoneal injection of phenylhydrazine (PHZ; $100 \mathrm{mg} / \mathrm{kg}$ ). $P<0.0001$, log-rank test; 1 of 2 independent experiments is shown. (B) ROS measurement in WT and Foxo3 ${ }^{--}$erythrocytes (note absence of any exogenously added peroxide). (C) Histogram of results from $\mathbf{B}$ (mean \pm SEM, $n=6$; $\left.{ }^{\star *} P<0.02\right)$. (D) Protein oxidation in erythrocytes. DNPH, 2,4-dinitrophenylhydrazine. (E) Quantitative analysis of the oxidative status of WT ( $n=3$ ) and Foxo3 $^{--}(n=4)$ erythrocytes by comparison of signal intensity of lanes in $\mathbf{D}$ using BandScan software version 4.5 (Glyco). ${ }^{*} P<0.05$, Student's $t$ test.

corrected the reticulocyte index in these mice (Figure 3B). After 5 weeks of treatment, NAC had some negative impact on the lifespan of wild-type cells (Figure 3A). Since in these studies the NAC treatment in terms of dose or frequency of administration was not optimized, we suspect that a prolonged chronic high dose NAC treatment might have adversely affected wild-type erythrocytes (33), as supported by our in vitro studies (Supplemental Figure 1B).

Foxo3 is highly expressed in hematopoietic organs and is activated during in vivo maturation of erythroid cells. These results indicated that Foxo3 may be directly involved in the regulation of oxidative stress in erythroid cells. To further investigate the function of Foxo3 in primary cells, we investigated the expression and transcriptional activity of Foxo3 during normal erythropoiesis. In these studies, E14.5 fetal livers that are highly enriched for post-progenitor erythroblasts (over $85 \%$ were TER $119^{+}$cells) were used.

Using quantitative real-time RT-PCR (QRT-PCR), Foxo3 was found to be abundantly expressed in the bone marrow and fetal liver (Figure 4, A and B). In addition, Foxo3 was the most highly expressed FoxO in erythroid cells (Supplemental Figure 1, C and D).
Foxo3 transcript (Figure 4C and Supplemental Figure 1C) and protein expression (Figure 4D) were gradually upregulated in freshly isolated subpopulations of fetal liver erythroid cells with increasing degree of maturation (34).

As anticipated, maturation of post-progenitor erythroid cells was accompanied by upregulation of expression of erythroidspecific genes $\alpha$ - and $\beta$-globin (35), erythroid Kruppel-like factor (EKLF, also known as Klf1), and Gata1 as well as downregulation of Gata2 transcription factors (Figure $4 \mathrm{C})$. The cyclin-dependent kinase (CDK) inhibitor 1B Cdkn1b ( $\left.227^{K I P 1}\right)$, a known transcriptional target of Foxo3 (9), is upregulated during erythroid maturation (36). Interestingly, the correlation between the patterns of Cdkn1b $\left(p 27^{\text {KIP1 }}\right)$ and Foxo3 expression suggest that Foxo3 may have a role in regulating $\mathrm{p} 27^{\mathrm{KIP} 1}$ expression in erythroid cells (Figure 4C). These studies (Figure 4, C and D) suggest that Foxo3 may be functional and transcriptionally active during maturation of primary erythroid precursor cells.

Expression of EpoR is the highest on mature erythroid progenitors colony forming unit-erythroid (CFU-erythroid) and is down- 


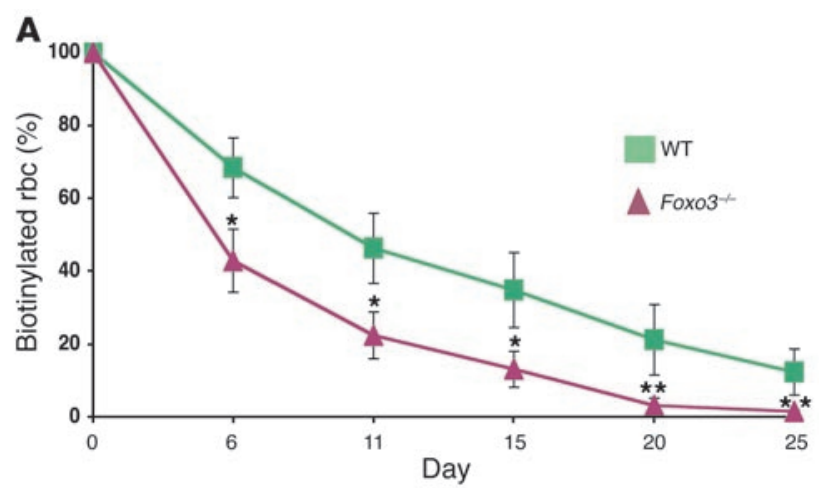

B

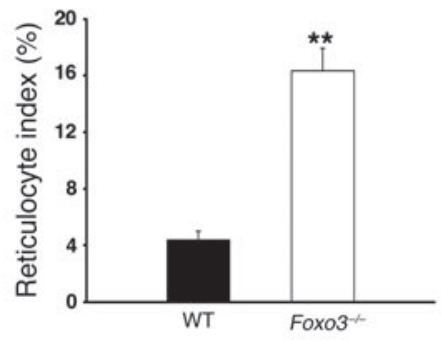

regulated thereafter with maturation; EpoR expression becomes very low on erythroid precursor cells $(37,38)$. Since the function of FoxO is negatively regulated by EpoR signaling and only nuclear FoxO is active (12), we hypothesized that nuclear localization and activity of Foxo3 may increase in erythroid precursors as cells lose the expression of EpoR and the sensitivity to EpoR signaling.

Using the subcellular localization of Foxo3 as a surrogate assay for the activity of Foxo3, we found that Foxo3 translocates to the nucleus in CD71+TER $119^{+}$cells and subsequent precursors during erythroid progenitor maturation (Figure 5A). Mature erythroid cells were 3 times more likely than their progenitors to express a nuclear Foxo3 (Figure 5B). To ensure that Foxo3 was detected in nucleated cells (Figure 5A), we used DRAQ5, a cell-permeable dye with high affinity for binding DNA in living and fixed cells. Foxo3 colocalization with DRAQ5 was over 2-fold in freshly isolated live, nucleated DRAQ5 ${ }^{+}$CD71-TER119 ${ }^{+}$cells enriched in mature normoblasts as compared with DRAQ5 ${ }^{+}$CD71-TER119- cells enriched in progenitors (Figure 5, C and D). These findings suggest that transcriptional activity of Foxo3 increases during in vivo maturation of primary erythroid precursors.

We next investigated more directly the transcriptional activity of endogenous Foxo3 using reporter gene assays in a previously established ex vivo differentiation system $(28,39)$ and a newly established transfection system of primary fetal liver erythroid cells (see Methods). Fetal liver erythroid subpopulations (as shown

Figure 3

ROS induced shortened erythrocyte lifespan in Foxo3-1- mice. (A) In vivo NAC therapy improves the lifespan of erythrocytes in Foxo3-1mice. WT and $\mathrm{FOxO}^{-/-}$mice were treated intraperitoneally with NAC $(100 \mathrm{mg} / \mathrm{kg})$ or PBS 3 times a week; after 3 weeks, erythrocytes were biotinylated in vivo and erythrocyte lifespan measured as in Figure 2, while NAC (or PBS) treatment continued for another 3 weeks. (B) Reticulocyte index was measured after 6 weeks in all mice. One of 2 independent experiments is shown. Results represent mean \pm SEM, $n=6$ per group; ${ }^{*} P<0.05$.

\section{Figure 2}

Reduced erythrocyte lifespan in Foxo3 $3^{-1-}$ mice. (A) Erythrocyte lifespan was measured by in vivo biotin labeling. At day $0,95 \%$ of erythrocytes were labeled. Animals were bled every 5 days, and the surviving labeled cells were analyzed by flow cytometry. (B) Reticulocyte index was measured in Foxo3 ${ }^{-/-}$and WT mice. Results represent mean \pm SEM; $n=6$ in each group. ${ }^{*} P<0.05,{ }^{* \star} P<0.01$, Student's $t$ test.

in Figure 4C) were transfected with luciferase reporters, and transcriptional activity was measured during ex vivo erythroid maturation (Figure 5, E and F).

The activity of endogenous Foxo3, using luciferase reporter containing 5 tandem repeat FoxO binding sites, was shown to be significantly upregulated more than 8-fold during 36 hours of ex vivo maturation of both early erythroid precursors (mostly CD71+TER119- proerythroblasts, which became CD71 $1^{+}$TER $119^{+}$) and intermediate erythroid precursors $\left(\mathrm{CD} 71^{+}\right.$TER $119^{+}$, which became CD71 ${ }^{\text {lo }}$ TER $\left.119^{+}\right)$compared with the mutant reporter lacking the Foxo3 binding site (Figure 5E, top panel). This FoxO reporter was not activated in Foxo3-deficient cells (Supplemental Figure 1E). These results suggest that Foxo3 activity is upregulated in intermediate erythroid precursors $\left(\mathrm{CD} 71^{+}\right.$TER $\left.119^{+}\right)$and stays on during their maturation. The strong increase in the activity of the erythroid transcription factor EKLF supports maturation stage of cells (Figure 5E, top panel). Given the expression pattern of Foxo1 and Foxo4, most if not all of the detected FoxO activity can be attributed to Foxo3 (Supplemental Figure 1C).

Consequently, we asked whether Foxo3 activates the transcription of antioxidant enzymes. The promoter of human catalase that is the most highly expressed antioxidant enzyme in erythroid cells contains several forkhead binding sites (8). Catalase reporter activity was highly upregulated in maturing erythroid cells transfected
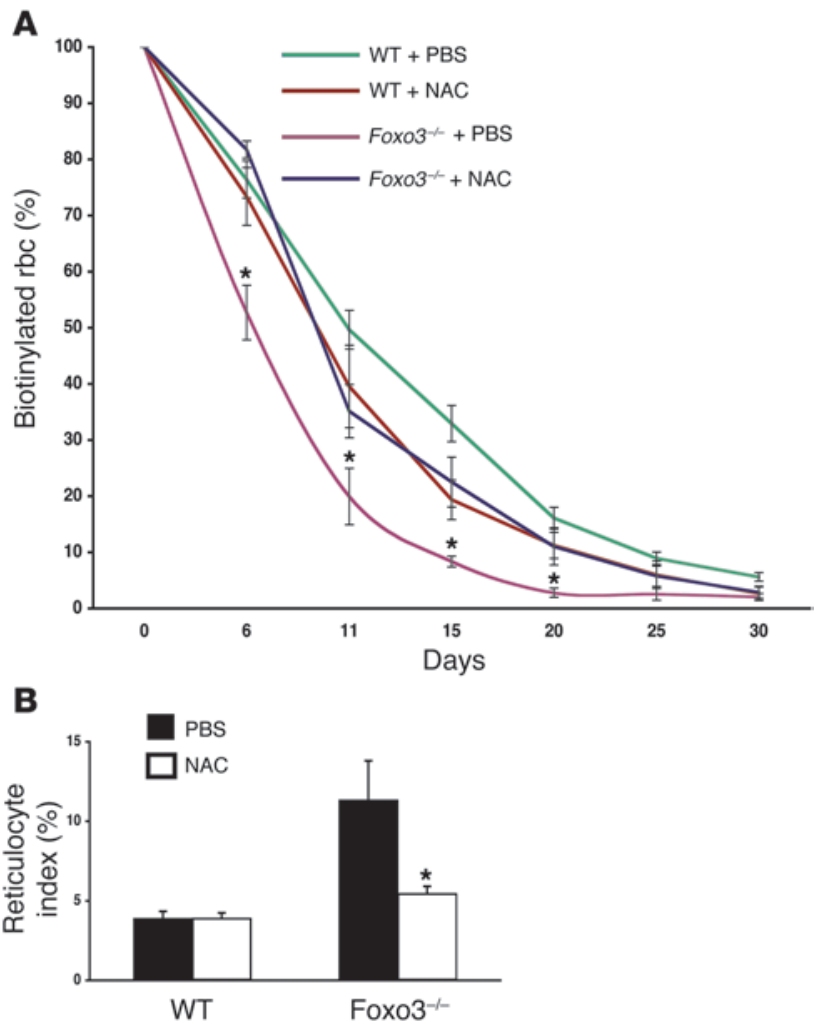
$\mathbf{A}$

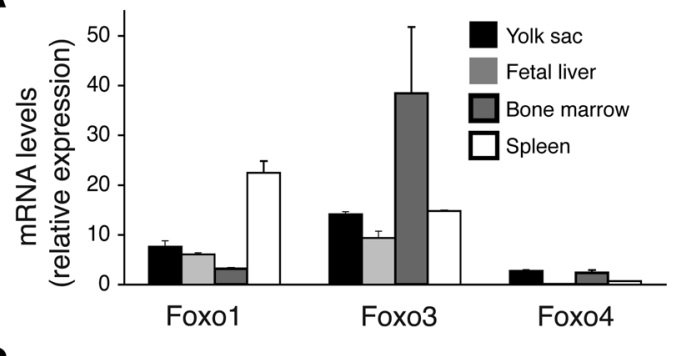

C

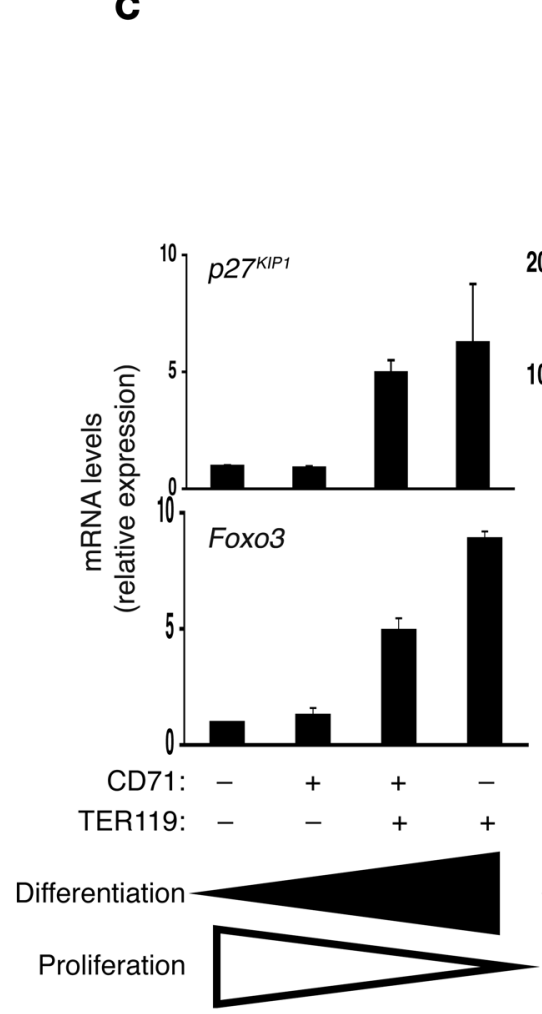

B

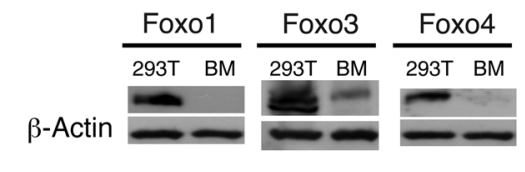

D

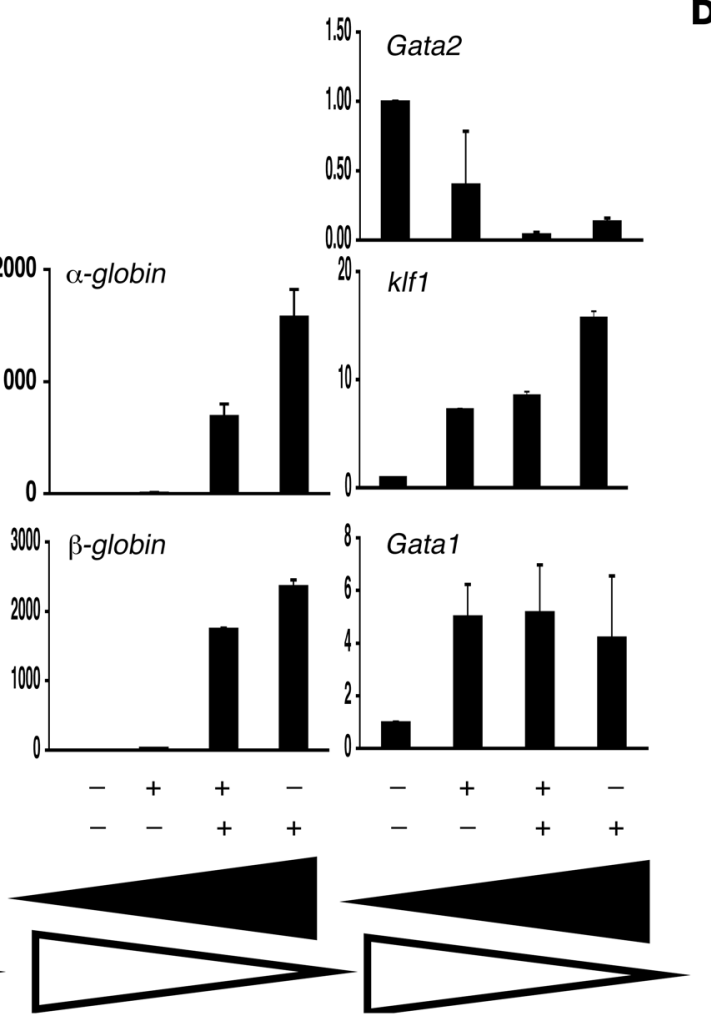

Figure 4

Foxo3 expression is upregulated during erythroblast maturation. (A) QRT-PCR analysis of Foxo1, Foxo3, and Foxo4 in embryonic and adult hematopoietic organs. Shown are representative results from 3 independent experiments performed in duplicate. (B) Western blot analysis of endogenous FoxO protein expression in the bone marrow. Protein lysates of HEK293T cells overexpressing FOXO1, FOXO3a, and FOXO4 cDNAs were used as positive controls. (C) QRT-PCR analysis of indicated transcripts from subpopulations of fetal liver isolated by flow cytometry according to their CD71 and TER119 expression. Note that expression of Foxo3 is the highest in the most mature erythroid cell subpopulation (CD71-TER119+). Representative results from 3 independent experiments performed in triplicate are shown as mean \pm SEM. Cells differentiate from a double CD71-TER119- cell subpopulation enriched in hematopoietic progenitors, including erythroid progenitors, to CD71+TER119- cells containing mostly proerythroblasts and basophilic erythroblasts, then to CD71+TER119+ cells enriched for basophilic/polychromatophilic erythroblasts, and finally to CD71-TER119+ cells consisting mostly of normoblasts. (D) Western blot analysis of Foxo3 in subpopulations of fetal liver enriched for progenitors (TER119-) and for erythroid precursors (TER119+, erythroblasts) using anti-FOXO3a antibody. Anti-GATA-1 (N6) and anti- $\beta$-actin antibodies were used as controls.

with a catalase reporter containing forkhead binding sites and not its mutant (Figure 5E, bottom panel; $P<0.001$ ).

The mitochondrial SOD2 is another important antioxidant enzyme in erythroid cells whose promoter contains 2 functional Foxo3 binding sites (7). Enhanced SOD2 reporter activity was detected during ex vivo maturation $(29,39)$ of erythroid progenitors at 48 hours (Figure 5F; $P<0.001$ ). This activity was significantly reduced in cells transfected with the reporter containing mutant Foxo3 binding sites (Figure 5F). These combined findings suggest that Foxo3 becomes nuclear and transcriptionally active at a stage of intermediary maturation of erythroid precursor cells
$\left(\mathrm{CD} 71^{+} \mathrm{TER} 119^{+}\right)$and regulates expression of some important antioxidant enzymes in erythroid cells.

Foxo3 coordinates proliferation and differentiation of erythroid cells by a ROS-mediated mechanism. In further support of the physiological function of Foxo3 in erythropoiesis, we found that the rate of maturation of bone marrow Foxo3 $3^{-/-}$erythroid cells was significantly reduced (2-fold less relative mature Foxo3 $3^{-/}$as compared with wildtype erythroid cells; Figure 6, A and B) and was accompanied by an increase in the size of the CD71+TER $119^{+}$erythroid precursor population. To investigate the mechanism of this reduction, we analyzed the cell cycle distribution of erythroid precursor cells that were at 
A

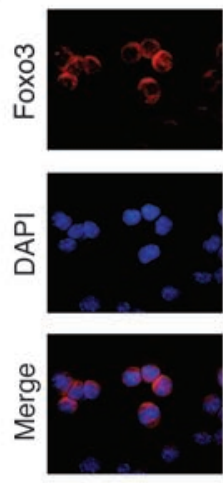

CD71:

TER119: -

Lanes: 1

Differentiation

Proliferation

C
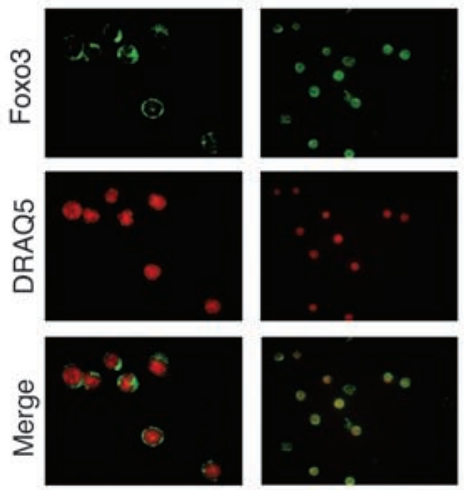

CD71:

TER119:
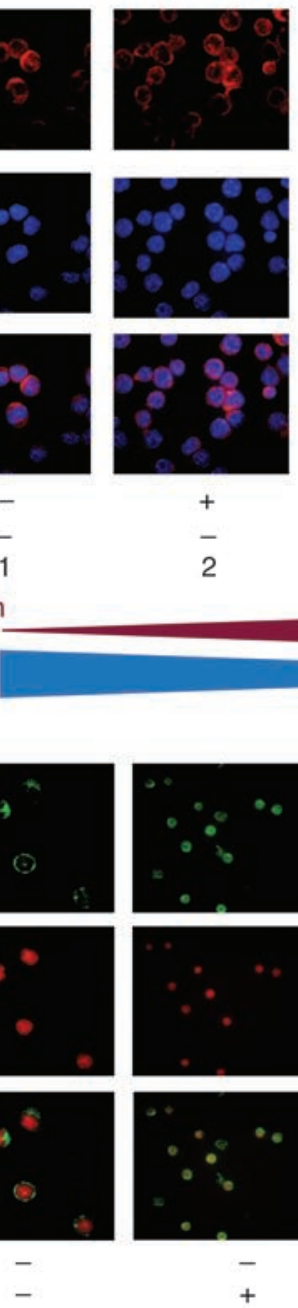

$+$

2
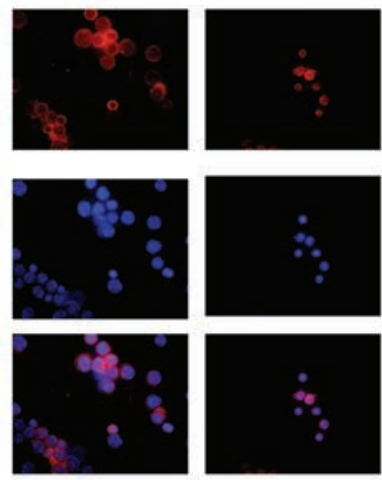

$+\quad+$

+
+
3

$+$

B

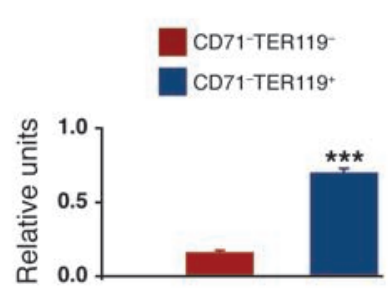

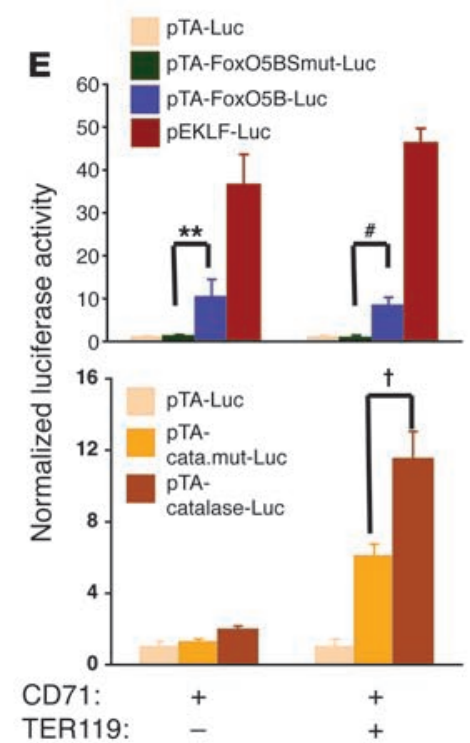

D
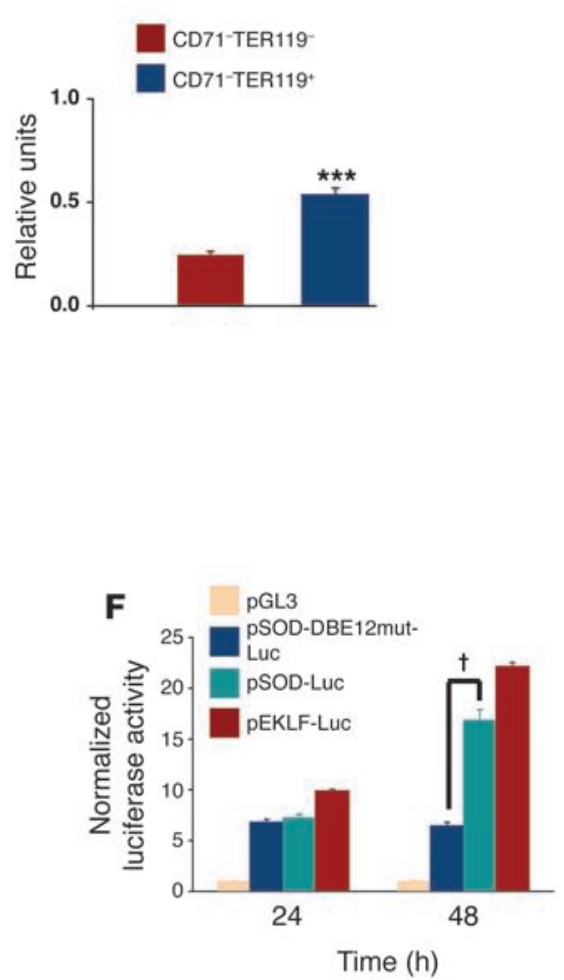

\section{Figure 5}

Foxo3 translocates to the nucleus and regulates transcription of anti-oxidant enzymes in primary fetal liver erythroblasts. (A) Immunofluorescence staining of Foxo3 (red) in freshly isolated E14.5 fetal liver erythroid subpopulations using antiFOXO3a antibody was performed and samples were counterstained with nuclear DAPI (blue). (B) Quantification of nuclear Foxo3 in CD71TER119- cells as compared with CD71-TER119+ cells. Data were analyzed from an average of 50 cells from each subpopulation in A. (C) Foxo3 expression was investigated in nucleated, deep red fluorescing agent (DRAQ5) positive cells. DRAQ5+CD71-TER119- and DRAQ5+CD71TER119+ cells were FACS sorted from E14.5 fetal liver and subjected to immunofluorescence staining using anti-FOXO3a antibody (green). (D) Quantification of results from C is shown for at least 50 cells from each subpopulation as mean \pm SEM. ${ }^{* * *} P<0.001$, Student's $t$ test. (E) FACS-sorted erythroid precursor subpopulations CD71+TER119- and CD71+'TER119+ cells were transfected with synthetic reporter containing 5 tandem repeat FoxO binding site (pTAFoxO5BS-Luc) or mutant (pTA-FoxO5BSmutLuc) (top panel), or a catalase luciferase reporter containing 2 FoxO binding sites (pTA-cata. mut-Luc) or mutant (pTA-catalase-Luc) (bottom panel), then cultured as previously described in the presence of Epo $(2 \mathrm{U} / \mathrm{ml})(28,39)$, and luciferase activity was analyzed after 36 hours. EKLF reporter ( $p E K L F-L u c)$ (56) was used as a positive control. Data representative of normalized results from at least 4 independent experiments performed in triplicate are shown as mean \pm SEM. ${ }^{* *} P<0.01 ;{ }^{\dagger} P<0.002 ;{ }^{\#} P<0.0001$. (F) Transcriptional activity of Foxo3 during maturation of fetal liver erythroid precursors. TER119fetal liver cells (enriched in progenitors) were transiently transfected with an empty luciferase reporter (pGL-3) or a SOD2 luciferase reporter containing 2 FoxO binding sites (pSod-Luc) or its mutant (pSOD-DBE12mut-Luc) and cultured as described above, and luciferase activity was determined at 24 and 48 hours. Results are shown as mean \pm SEM; $n=4$ for each group. different stages of differentiation (Figure 6, A and C). Given that one of the functions of Foxo3 is to induce mitotic arrest by repressing the entry into the $\mathrm{S}$ phase and Foxo 3 is transcriptionally active in wild-type erythroid precursors (CD71+TER119+ cells; Figure 5), we suspected that the normal function of Foxo3 during maturation is to inhibit the cell cycle progression of this specific population of ery- 
A

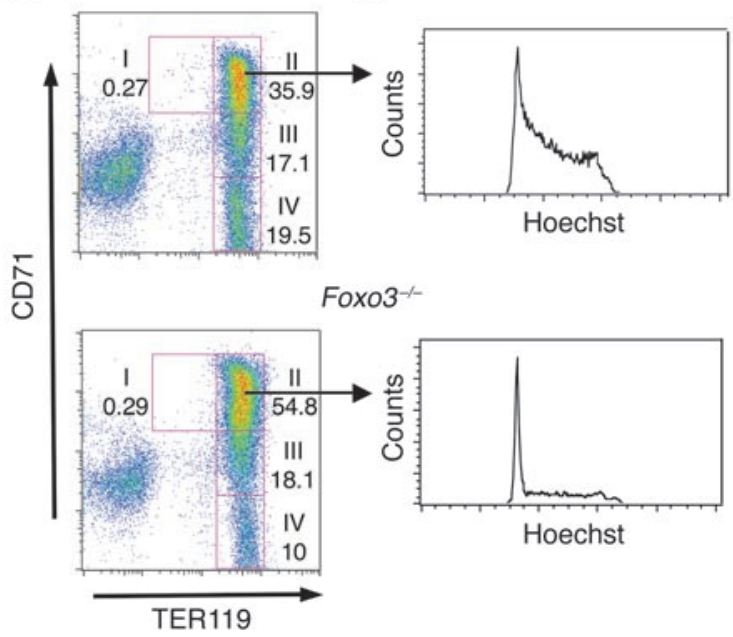

B

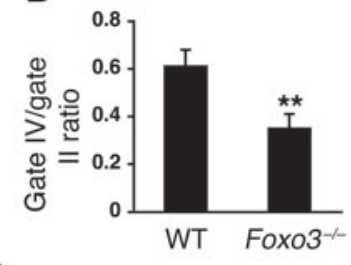

C

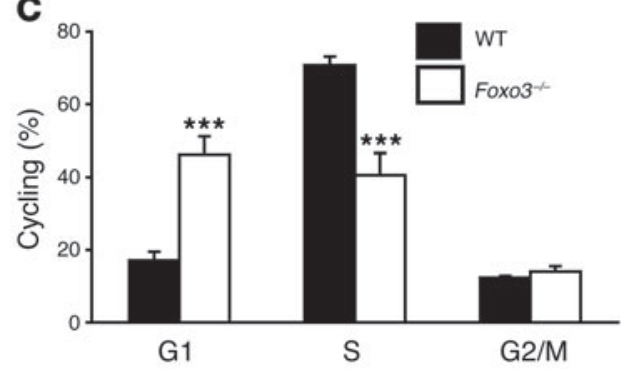

Figure 6

Mitotic arrest in Foxo3--- erythroid precursor cells. (A) Left: Flow cytometry analysis of live WT and Foxo3-- bone marrow erythroid precursors according to their expression of CD71 and TER119. Gates I to IV depict erythroid cell populations with an increasing degree of maturation. Numbers by gates represent percentage of distribution of cells within that gate. Note decrease of $\mathrm{FoxO}^{-1-}$ mature erythroid cells (gate IV) and increase of $\mathrm{FoxO}^{-1-}$ precursors in gate II as compared with WT (representative flow cytometry analysis of 9 experiments is shown). Right: Representative analysis of cell cycle distribution in CD71+TER119+ cells (gate II) from 6 independent experiments. (B) Ratio of gate IV to gate II from $\mathbf{A}\left(n=9\right.$, mean $\pm \mathrm{SEM} ;{ }^{* \star} P<0.01$, Student's $t$ test). (C) Cell cycle analysis of cells in gate II from left panel of $\mathbf{A}\left(n=6\right.$, mean \pm SEM; ${ }^{* \star *} P<0.001$, Student's $t$ test) is shown. WT and Foxo3-deficient CD71 ${ }^{+}$TER119+ (gate II) bone marrow erythroid cells were FACS sorted and analyzed for cell cycle distribution using Hoechst (A, right panel, and C). Percentage of cells in G1, S, and G2/M phases of cell cycle are shown ( $n=6$, mean $\pm \mathrm{SEM}$; ${ }^{* \star *} P<0.001$, Student's $t$ test). No significant difference was found in the analysis of cell cycle of other subpopulations.

throid precursor (CD71+TER119+) cells. Such a contention would predict an increase in proliferation of Foxo3-deficient erythroid precursor cells, which could delay their maturation.

To our surprise however, loss of Foxo3 resulted in a significant fraction of Foxo3-deficient erythroid precursors, most of which were found in the G1 phase of the cell cycle, displaying mitotic arrest (Figure 6, A and C). The alteration in cycling of erythroblasts was not observed in other erythroid subpopulations. These highly unexpected findings indicate that functional Foxo3 is specifically required for normal regulation of the cell cycle during erythroid cell maturation. In addition, these results suggested that the decreased rate of maturation may not provide sufficient reticulocytosis in case of a massive hemolysis.

To elucidate the potential mechanism of alteration of the Foxo3deficient cell cycle, the transcript levels of candidate molecules previously implicated in erythroid cell cycle and/or known to be transcriptional targets of Foxo3 were assessed. The expression of the CDK inhibitor $2^{2} 7^{K I P 1}$ and cyclin G2 (Ccng2) transcripts, both downstream targets of Foxo3 $(9,10,40)$, was significantly reduced in Foxo $3^{-/-}$erythroid subpopulations (Figure 7A), suggesting that the expression pattern of $\mathrm{p} 27^{\mathrm{KIP} 1}$ or cyclin G2 can not explain the alteration of cell cycle observed in Foxo3 $3^{-/-}$erythroid precursors and that these genes are likely to be transcriptional targets of Foxo3 in erythroid precursor cells.

Unexpectedly however, the expression of another CDK inhibitor, $p 21^{C I P 1 / W A F 1 / S d i 1}(\mathrm{Cdkn} 1 \mathrm{a})$, which, similar to $\mathrm{p} 27^{\mathrm{KIP} 1}$, is an inhibitor of the transition from the G1 to the $\mathrm{S}$ phase of cell cycle and a known downstream target of Foxo3 (41), was significantly upregulated in Foxo3-deficient cells (Figure 7A). targets of 53 activated in response to are known downstream (Figure 7C). These results further support that activation of p53 in Foxo3-deficient erythroid precursor cells (CD71+TER119+) is in response to an oxidative stress condition. Consistent with this prediction, transcription of heme oxygenase 1 (HO-1), which is known to be associated with oxidative stress (46), and not its closely related molecule HO-2 was also upregulated (Figure 7C).

Together these findings strongly suggest that upregulation of $\mathrm{p} 21^{\mathrm{CIP} 1 / \mathrm{WAF} / \mathrm{Sdil}}$ and the mitotic arrest observed in erythroid precursors is in response to a state of oxidative stress (47). To assess this we tested the effect of ROS scavengers on $\mathrm{p} 21^{\mathrm{CIP} 1 / \mathrm{WAF} 1 / \mathrm{Sdi} 1}$ expression. As shown in Figure 8, the level of $221^{\text {CIP1/WAFI/Sdil was }}$ significantly higher after 36 hours in a population of cultured Foxo3-deficient bone marrow cells as compared with a population of wild-type bone marrow cells that were highly enriched in progenitors and depleted of mature cells. Incubation with ROS scavenger NAC resulted in significant downregulation of $p 21^{C I P 1 / W A F 1 / S d i 1}$ in Foxo3-deficient cells (Figure 8).

Consistent with the results discussed above, the expression of several ROS-scavenging enzymes was significantly downregulated in Foxo3-deficient erythroid precursor cells (TER119+; Figure 9). Inhibition of expression of catalase and SOD2 in Foxo3-deficient erythroid precursor cells further supports a role for Foxo3 in the regulation of these enzymes in erythroid precursor cells. In addition, we found that expression of glutathione peroxidase, which has an important antioxidant function (26), and the cytoplasmic SOD1 is strongly repressed in Foxo3-deficient erythroid precursor cells. Thus the state of oxidative stress in erythroid cells is likely due to downregulation of antioxidant enzymes in Foxo3-mutant erythroid precursor cells. 

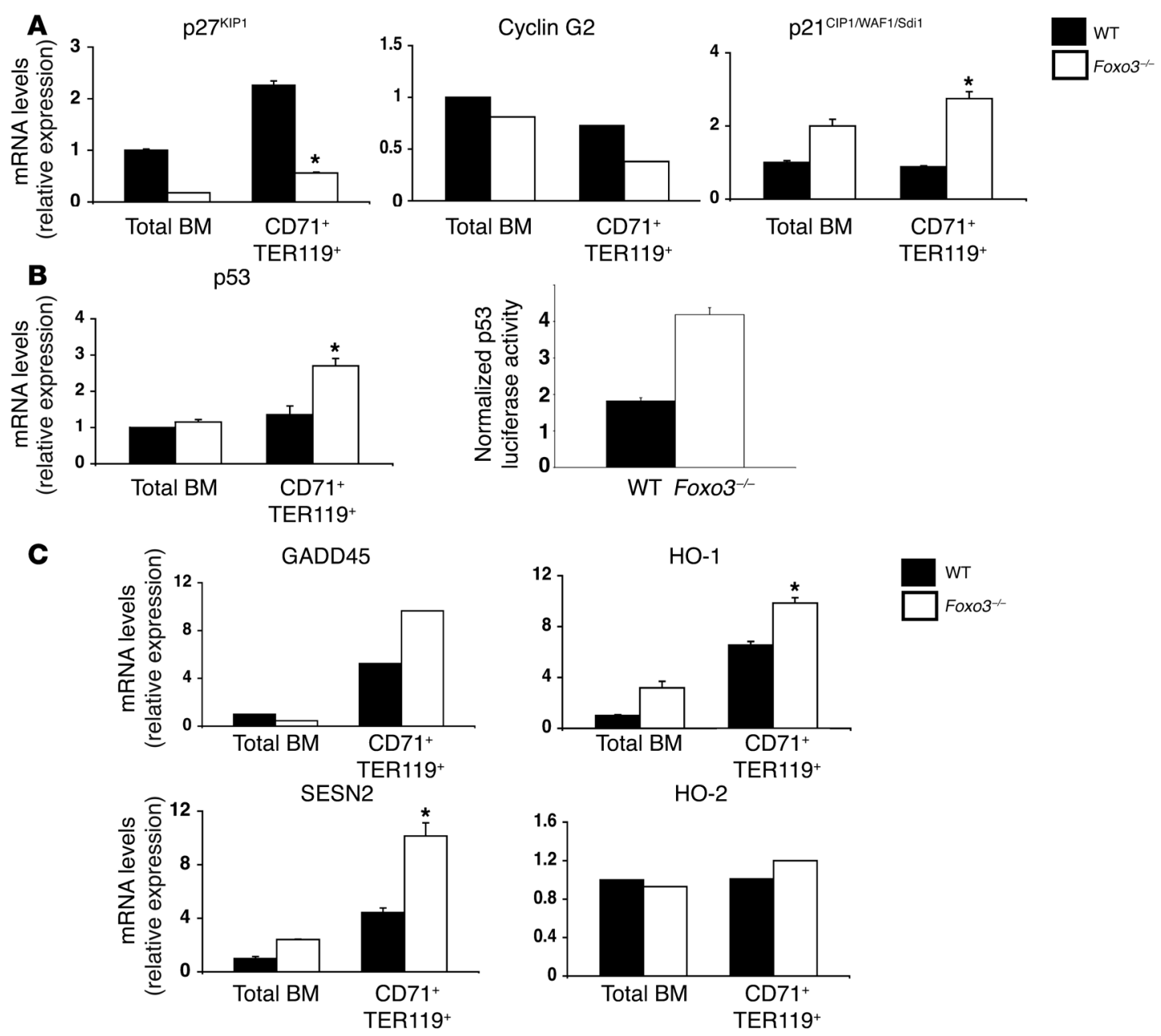

\section{Figure 7}

State of oxidative stress in Foxo3-deficient erythroblasts. (A) QRT-PCR analysis of cell cycle regulator genes in freshly isolated FACS-sorted erythroblasts at an intermediary (CD71+TER119+) stage of differentiation from wild-type and Foxo3-deficient bone marrow cells. Note upregulation of p21 CIP1/WAF1/Sdi1 in intermediary erythroblasts (CD71+TER119+ cells are the same as those shown in gate II of Figure 6A). (B) Left: QRT-PCR analysis of p53 expression in the same cells as in A. Right: p53 reporter luciferase activity in WT and Foxo3-deficient bone marrow TER119- cells 36 hours after transfection and in vitro culture as in Figure 5, E and F. Representative of 2 independent experiments. (C) QRT-PCR analysis of antioxidant genes in the same populations as in A. Note upregulation of p53 downstream antioxidant targets GADD45 and SESN2. Representative of 4 independent experiments performed in duplicate or more. ${ }^{*} P<0.05$, Student's $t$ test.

Finally, to further elucidate the cause of death of Foxo3-deficient mice subjected to phenylhydrazine treatment, Foxo $3^{-/-}$and wild-type mice were treated with PBS or $100 \mathrm{mg} / \mathrm{kg}$ phenylhydrazine and sacrificed 24 hours later for analysis. In response to phenylhydrazine treatment, the numbers of Foxo3-deficient progenitors produced in the bone marrow and spleen were not significantly different from those of wild-type mice (data not shown). However, the relative frequency of Foxo3-deficient mature erythroid cells produced in response to the treatment was extremely low (less than 1\%) and over one-third of that of wild-type bone marrow (Figure 10A). As anticipated at 24 hours, the main impact of the treatment (48) was on the bone marrow, although similar results were also observed in the Foxo3 ${ }^{-1}$ spleen and blood (Figure 10A). These data may suggest that spleen's environment is more protective against oxidative stress. In agreement with these results, ROS were substantially more concentrated in the Foxo3-deficient bone marrow erythroid cells (TER119+) as compared to wild type controls (Figure 10B). It is noteworthy that at the steady state, ROS concentration in Foxo3-deficient erythroid precursor cells (TER119+) in the bone marrow was as elevated as in wild-type mice treated with phenylhydrazine (Figure 10B; compare PBS-treated $\mathrm{Foxo3}^{-/-}$with phenylhydrazine-treated wild-type cells in the bone marrow). These results suggest that at the steady state, Foxo3-deficient erythroid cells are chronically subjected to the same levels of ROS as the levels induced by phenylhydrazine in wild-type erythroid precursors (Figure 10B). A correlation between G1 arrest and ROS concentration was also observed in treated animals (data not shown). Together these results strongly suggest that the death induced by phenylhydrazine treatment in Foxo3-deficient mice is likely due to an excessive destruction of circulating erythrocytes coupled with a significant ROS-induced mitotic arrest (see Figures 6 and 8), especially in the bone marrow, and a significantly decreased number of mature erythroid cells due to loss of Foxo3 that impedes a timely hematopoietic response. 


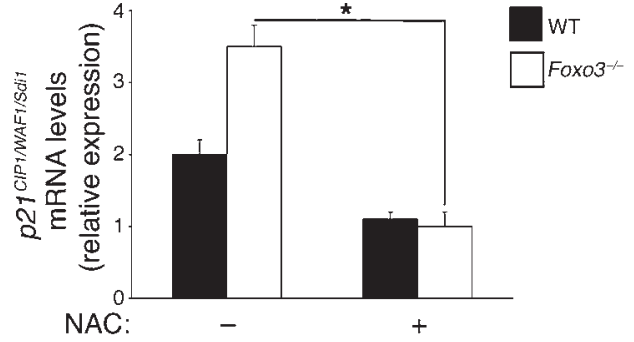

Figure 8

ROS-induced upregulation of $p 21_{\text {CIP1/WAF1/Sdi1. TER119- bone marrow }}$ cells were cultured with or without $100 \mu \mathrm{M} N A C$, and the expression of p21 ${ }^{\text {CIP1/WAF1/Sdi1 }}$ was measured by QRT-PCR after 36 hours. ${ }^{*} P<0.05$ Student's $t$ test.

\section{Discussion}

The combined data presented here provide a model in which activation of Foxo3 in erythroid precursors is essential for their optimal cell cycle regulation and maturation, for red blood cell lifespan and for erythrocyte resistance to deleterious effects of oxygen radicals. Although anomalies of Foxo3-deficient erythropoiesis are compatible with life at the steady state, mice died rapidly when exposed to induced oxidative stress as a result of enhanced destruction of erythrocytes and lack of sufficient production of mature erythrocytes due to a blunted response. Our study also provides the first evidence for in vivo requirement for Foxo 3 regulation of oxidative stress in mammals. In addition, these results demonstrate that the potential redundancy among FoxO proteins does not preclude the requirement for Foxo 3 in antioxidative stress response and optimal erythropoiesis.

In addition to hemoglobin toxicity, erythroid cells are exposed to free iron during both mitochondrial insertion of ferrous iron into heme in precursors and release of iron from hemoglobin in mature cells, which results in deleterious damage catalyzed by iron through generation of hydroxyl radicals by means of Fenton reaction (23). Defenses against ROS include enzymes such as SOD, which converts superoxide to hydrogen peroxide; catalase and glutathione peroxidase, which convert hydrogen peroxide to water; non enzymatic scavengers such as glutathione; and a family of peroxiredoxin antioxidant proteins (26).

In agreement with ROS regulation of erythrocyte lifespan (27), expression of several antioxidant enzymes, including glutathione peroxidase and SOD1, that were not previously known to be regulated by FoxO, was significantly reduced in Foxo3-deficient erythroid cells (Figure 9). It is noteworthy that we found conserved FoxO TTGTTTAC binding sites (49) in both mouse and human glutathione peroxidase promoter sequences, suggesting that Foxo3 may also directly regulate transcription of glutathione peroxidase. In addition, genetic deletion of SOD2 has been directly implicated in a ROS-mediated shortened erythrocyte lifespan (24), suggesting that the phenotype observed in Foxo3-deficient mice may be a result of the relative defect in SOD2 transcription in erythroid precursors.

Foxo3-deficient erythroid precursor cells exhibit an unanticipated mitotic arrest that is in contrast to the function of Foxo3 as an inhibitor of cell cycle. Although the sequence of events leading to the growth arrest in these cells is not known, several features of $\mathrm{Foxo3}^{-/-}$cell cycle defect are notable. First, the inhibition of G1/S transition of cell cycle was only seen in a popu- lation of highly cycling erythroid precursors $\left(\mathrm{CD} 71^{+}\right.$TER $\left.119^{+}\right)$ that is known to be activated in response to stress $(50,51)$ (Figure 6C) and that accumulates hemoglobin while losing response to Epo due to a gradual loss of EpoR expression (37, 38). Second, the inhibition of G1/S transition was characterized by a stress response condition manifested by a ROS-mediated upregulation of CDK inhibitor $p 21^{C I P 1 / W A F 1 / S d i 1}$ (Figure 8) and several antioxidant response genes (45), targets of p53 tumor suppressor (Figure 7C). Third, p53 was activated in erythroid precursor $\left(\right.$ CD71 $1^{+}$TER $119^{+}$) cells (Figure 7 ). While upregulation of $\mathrm{p} 21^{\mathrm{CIP} 1 / \mathrm{WAF} 1 / \mathrm{Sdi1}}$ is implicated in normal maturation of erythroid precursors (52), a premature upregulation of $\mathrm{p} 21^{\mathrm{CIP} 1 / \mathrm{WAF} 1 / \mathrm{Sdi} 1}$ during stress-induced expansion of Foxo3-deficient erythroid precursors would lead to a premature cell cycle exit resulting in a significant decrease of the number of mature cells. Although we did not detect any apoptosis in freshly isolated Foxo3-deficient mature erythroid cells, we can not rule out additional loss through apoptosis, specifically since Foxo3-null bone marrow cells are highly prone to apoptotic death once in culture (D. Marinkovic and S. Ghaffari, unpublished observations). Together these results strongly suggest that hindrance in maturation and response to induced oxidative stress in Foxo3-deficient erythroid precursors is a major contributor to death of animals when exposed to acute exacerbated oxidative stress.

Inhibition of FOXO3a is important for Epo-mediated survival of cultured erythroid cells $(18,19,53)$. As Foxo3 is mostly cytoplasmic and presumably inhibited in erythroid progenitors (Figure 5, A-D), its function may be limited to inducing apoptosis in dividing progenitor cells subjected to cytokine deprivation, in agreement with the context-dependent function of FoxO (6). Although the mechanism of nuclear translocation and activation of Foxo3 in erythroid precursors (CD71+TER $119^{+}$) is unknown, it is plausible that downregulation of EpoR (i.e., inhibition of EpoR signaling) coupled with the accumulation of hemoglobin (i.e., enhanced ROS build-up) in these cells provides a signal for normal nuclear translocation and activation of Foxo3 to prevent further ROS accumulation and cellular damage (Figure 11).

Like p53, ataxia telangiectasia mutated (ATM) gene product is often activated as part of an antioxidant response (54). Given that the function of ATM in erythropoiesis is not known, we were surprised to find a significant and specific downregulation of ATM in Foxo3-deficient cells (see the relative expression

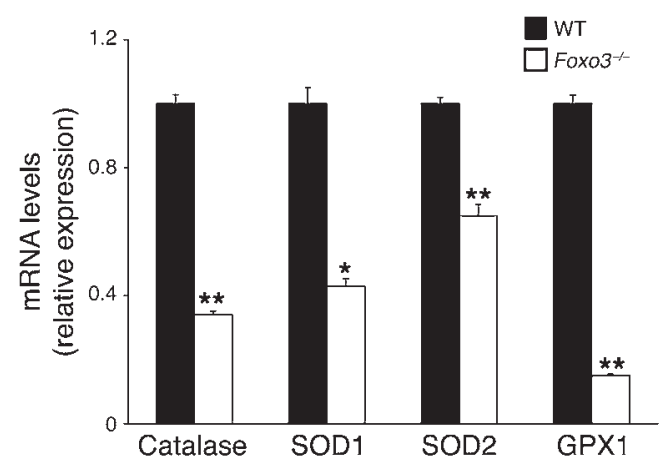

\section{Figure 9}

Fox03 is required for expression of antioxidant enzymes. QRTPCR analysis of antioxidant enzymes in bone marrow erythroblasts (TER119+) cells. GPX1, glutathione peroxidase 1. $n=3$; ${ }^{\star} P<0.05$, ${ }^{\star *} P<0.02$, Student's $t$ test. 


\section{WT
Foxo3 $^{-1}$}
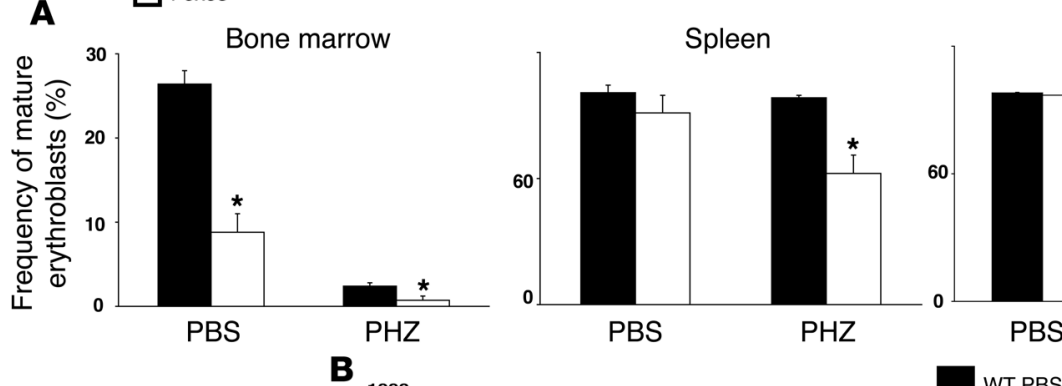

Blood

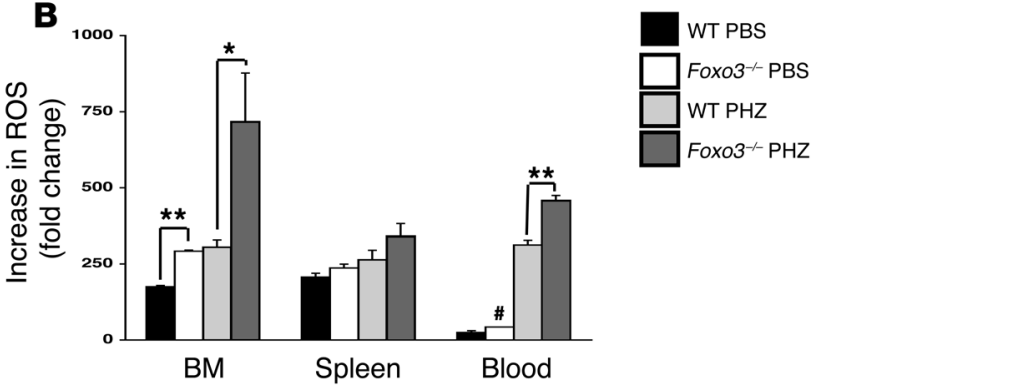

\section{Figure 10}

Defective post-progenitor erythroblast response to phenylhydrazine in $\mathrm{FoxO}^{-/-}$mice. WT and Foxo3-deficient mice were treated with phenylhydrazine $(100 \mathrm{mg} / \mathrm{kg})$ or PBS and sacrificed 24 hours later. (A) Flow cytometry analysis of relative frequency of mature erythroblasts in the bone marrow, spleen, and blood, calculated by the ratio of mature erythroblasts (CD71-TER119+) to total erythroblasts (CD71+TER119+ and CD71-TER119 $\left.{ }^{+}\right) . n=3$ in each group; ${ }^{*} P<0.05$, Student's $t$ test. (B) Measurement of ROS concentration in erythroblasts (TER119+ cells). " $P<0.01$; ${ }^{* *} P<0.001$. of ATM in the bone marrow as compared to in erythroid cells; Supplemental Figure 1F). Given the known association of ATM deletion with enhanced ROS concentration (2), these observations suggest that downregulation of ATM may participate in the molecular pathway regulating antioxidant signaling downstream of Foxo3 in erythroid cells.

Triple deletion of FoxO was recently shown to result in hematopoietic stem cell defect associated with high oxidative stress (55). Our results expand these findings and attribute an essential role to Foxo3 in the regulation of oxidative stress in erythroid cells. Given that expression of Foxo 4 and Foxo 1 transcripts is barely detectable in wild-type or Foxo3-deficient bone marrow erythroblasts (note the absence of upregulation of Foxo1 or Foxo4 transcripts in Foxo3-deficient TER119+ erythroblasts; Supplemental Figure 1D) and given that Foxo1 and Foxo 4 proteins were undetectable in bone marrow erythroid precursor cells (TER119 ${ }^{+}$erythroblasts; Supplemental Figure 1G), Foxo1 and Foxo 4 do not appear to be relevant to the Foxo3-deficient erythroid phenotype. Although we can not rule out the expression of functional Foxo1 and/or Foxo4 in erythroid cells, our data strongly suggest that it is unlikely these FoxO play a significant role in erythroid cell maturation (Figure 4, A and B, and Supplemental Figure 1, C, D, and G).

In addition to the erythroid phenotype described here, Foxo3deficient mice exhibit a global ovarian follicular activation leading to oocyte death and early depletion as well as a lymphoproliferation associated with activation of NF- $\mathrm{BB}(15-17)$. It is plausible that these phenotypes are also induced by enhanced oxidative stress. In particular, given the absence of evidence for direct regulation of elements of NF-אB pathway by Foxo3 (17), it

\section{Figure 11}

Model of the regulation of Foxo3 subcellular localization in erythropoiesis. Inhibition of EpoR signaling, which includes inhibition of the PI3-kinase/AKT pathway, as a result of downregulation of EpoR on erythroblasts, coupled with accumulation of hemoglobin, may result in ROS signaling, leading to Foxo3 nuclear localization and activation. is conceivable that activation of NF-кB in Foxo3-deficient T cells results from enhanced oxidative stress.

Finally, it is noteworthy that results presented here were obtained from young adult mice (8-12 weeks old). Given the correlation of oxygen radicals with aging (1), it is likely that this phenotype will be exacerbated in older Foxo3 $3^{-1-}$ mice.

The combined results presented here demonstrate that Foxo3 is a critical physiological regulator of oxidative stress in mammalian cells. These findings establish essential functions for Foxo3 in the regulation of cell cycle, maturation, lifespan, and resistance to oxidative stress during erythropoiesis. Whether activity of Foxo3 can be exploited to increase erythrocyte lifespan and maturation is of major interest.

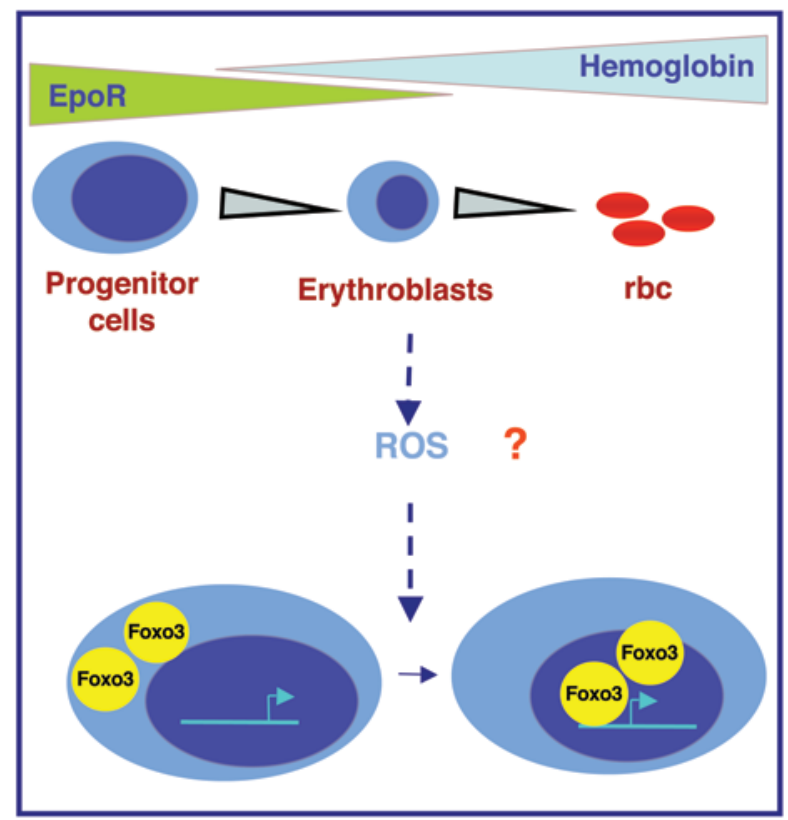




\section{Methods}

Mice. The generation and genotyping of mice was performed as previously described (15). Littermates (8 to 12 weeks old) of heterozygous intercrosses were used in all experiments. Protocols were approved by the Institutional Animal Care and Use Committee of Mount Sinai School of Medicine. Wildtype fetal liver cells were isolated from E14.5 C57BL/6 embryos (Jackson Laboratories) as previously described (28).

Hematological analysis. Blood samples were collected in 2 rounds from mouse tail vein in the presence of $25 \mathrm{mM}$ EDTA and analyzed using Bayer ADVIA 120 Hematology System. To measure erythrocyte lifespan, a total dose of $100 \mathrm{mg} / \mathrm{kg} \mathrm{N}$-hydroxysulfosuccinimide-biotin (EZ-Link; Pierce Biotechnology) was injected intravenously into mice. Biotin-labeled cells were revealed by Streptavidine-Cy-Chrom binding (BD Biosciences Pharmingen) (32). Number of survived biotin-labeled cells remaining in circulation was calculated as a percentage of positively biotinylated cells detected on day 1 after injection (95\%). For phenylhydrazine treatment, mice were injected intraperitoneally with phenylhydrazine hydrochloride (Acros Organics; Thermo Fisher Scientific); blood samples were collected every 2 to 3 days for measurement of hematocrit and reticulocyte levels.

Flow cytometry. Flow cytometry was performed at Mount Sinai Flow Cytometry Shared Research Facility as previously described (28).

Cell cycle analysis. Cells were incubated with $10^{6} / \mathrm{ml}$ Hoechst 33342 in prewarmed DMEM supplemented with 15\% FCS for 90 minutes, stained with antibodies, and analyzed immediately by FACS using FACSDiva software (version 5.0.2; BD Biosciences).

QRT-PCR. Total RNA was isolated using RNeasy Mini Kit (QIAGEN) according to the manufacturer's instructions. First-strand cDNA was synthesized with SuperScript First-Strand Synthesis System for RT-PCR kit using SuperScript II reverse transcriptase enzyme (Invitrogen), and QRT-PCR reactions were performed using SYBR Green JumpStart Taq ReadyMix (Sigma-Aldrich) in duplicates (LightCycler 2.0; Roche Diagnostics). Gene-specific primers were designed to span intron-exon boundary by Primer Express 2.0 (ABI) and then subjected to blast analysis to ensure the primer specificity. Relative quantification was achieved by normalization to endogenous $\beta$-actin. For primer sequences, see Supplemental Table 2 .

Measurement of intracellular ROS. Cells were washed and resuspended in pre-warmed PBS supplemented with $2 \%$ FCS and loaded with $5 \mu \mathrm{M}$ 5-(and 6-)-chloromethyl-2', $7^{\prime}$-dichlorodihydrofluorescein diacetate (CM- $\mathrm{H}_{2}$ DCFDA; Invitrogen) in the dark for 20 minutes at $37^{\circ} \mathrm{C}, 5 \% \mathrm{CO}_{2}$. The oxidative conversion of CM- $\mathrm{H}_{2} \mathrm{DCFDA}$ to its fluorescent product was measured immediately by flow cytometry.

Protein oxidation detection. Erythrocyte cell lysate proteins $(15 \mu \mathrm{g})$ were reacted with 2,4-dinitrophenylhydrazine to derivatize carbonyl groups formed through protein oxidation (OxyBlot Protein Oxidation Detection Kit; Chemicon International) to dinitrophenylhydrazone (DNP) and resolved by $12 \%$ SDS-PAGE and derivatized residues detected by rabbit anti-DNP antibody according to OxyBlot protocol. Band intensity was measured using BandScan software version 4.5 (Glyco).

Western blot analysis. Bone marrow cell lysates were prepared using lysis buffer (50 mM Tris-Cl, pH 6.8, 2\% SDS, 10\% glycerol, 10 mM DTT, protease inhibitor cocktail inhibitor tablets [Roche Diagnostics], $10 \mathrm{mM}$ PMSF, $10 \mathrm{mM}$ EDTA), and proteins were resolved by $10 \%$ SDS-PAGE, transferred to nitrocellulose membranes, and incubated with anti-FKHR/FOXO1 (Santa Cruz Biotechnology Inc.), anti-FKHRL1/FOXO3a (Millipore), or anti-AFX1/ FOXO4 (Santa Cruz Biotechnology Inc.) antibodies (1:250 dilution each).

Immunofluorescence staining. FACS-sorted subpopulations of E14.5 fetal liver cells were cytospun, fixed with $4 \%$ paraformaldehyde, and immunostained as described previously (12). Cells were permeabilized using $1 \%$ Triton X-100, 3\% BSA, and 1× PBS, incubated with anti-
FOXO3a antibody (1:500) in 1\% BSA for 1 hour, washed and stained with Alexa Fluor 594-conjugated anti-rabbit IgG antibody $(5 \mu \mathrm{g} / \mathrm{ml}$; Invitrogen), washed, and mounted with VECTASHIELD Mounting Medium with DAPI (Vector Laboratories) to stain the nucleus. Images were captured using Nikon Eclipse E600 microscope with $\times 630$ magnification and processed with Adobe Photoshop software version 7.0. Deep red fluorescing agent (DRAQ5) (Sigma-Aldrich) was used at 1:1,000 dilution for 5 minutes at room temperature prior to antibody staining and FACS sorting. Data was quantified using CoLocalizer Pro software (CoLocalization Research Software version 1.2) and the overlap coefficient according to Manders (57).

NAC treatment. NAC (Sigma-Aldrich) solution in PBS pH 7.4 $(100 \mathrm{mg} / \mathrm{kg}$ of body weight) was administered intraperitoneally to mice 3 times per week for the indicated time period (see Results).

Reporter gene assay. FACS-sorted fetal liver subpopulations were transiently transfected with $50 \mathrm{ng}$ of pRL-TK (Promega) alone or with pRL-TK and $2 \mu \mathrm{g}$ of one of the following: catalase luciferase reporter containing 2 FoxO binding sites (pTA-3179-Luc, also known as pTA-catalase-Luc) or mutant catalase reporter (pTA-1645-Luc, also known as PTA-cata.mut-Luc) (8); a synthetic reporter containing 5 tandem repeat FoxO binding sites (pTA-FoxO5BS-Luc) or mutant FoxO reporter (pTA-FoxO5BSmut-Luc); or an EKLF reporter (pEKLF-Luc, pHS2 $\beta$ Luc) (56), using Amaxa nucleofection technology (Program $\mathrm{X}-01,40 \%-70 \%$ transfection; Amaxa Inc.), seeded $\left(5 \times 10^{5}\right.$ cells $\left./ \mathrm{ml}\right)$ in triplicate as previously described (29) in the presence of Epo $(2 \mathrm{U} / \mathrm{ml})$, and analyzed for luciferase activity after 36 hours.

TER119- $\left(1 \times 10^{7}\right)$ cells were transiently transfected with pSOD-Luc $(7)$ or its mutant PSOD-DBE12mut-Luc (7) and both were cultured for either 24 or 48 hours, after which cells were lysed and luciferase activity measured. Oligonucleotides containing 5 tandem repeat FoxO binding sites or mutants were ligated to $\mathrm{PGL}-3$ vector.

Statistics. The unpaired 2-sample Student's $t$ test was used. A $P$ value of less than 0.05 was considered to be significant.

\section{Acknowledgments}

This work was supported by an American Cancer Society Research Scholarship (RSG LIB-110480) and a grant from the National Heart, Lung, and Blood Institute (HL076510-01) to S. Ghaffari. We thank Ron Dephino (Dana Farber Cancer Institute) for kindly providing $\mathrm{FoxO}^{+/-}$mice, Boudewijn Burgering (University Medical Centre Utrecht) for SOD2 reporters, Michael Greenberg and Anne Brunet (Harvard Medical School) for FOXO3a cDNA, Karen Arden (UCSD) and Bill Sellers (Dana Farber Cancer Institute) for FOXO1 and FOXO4 cDNAs, Toren Finkel and Shino Nemoto (NIH) for catalase reporters, Stuart Aaronson and James Bieker (Mount Sinai School of Medicine) for p53 and EKLF reporters, respectively, and James Evans (Massachusetts Institute of Technology) and Stuart Fraser (Mount Sinai School of Medicine) for advice about analysis of immunofluorescence data and DRAQ5, respectively. We also thank Anoushiravan Amini for mouse genotyping and Italas George and Marcos Grisotto for FACS sorting. We are grateful to Reshma Taneja and David Sternberg (Mount Sinai School of Medicine) for critically reading the manuscript.

Received for publication February 13, 2007, and accepted in revised form May 8, 2007.

Address correspondence to: Saghi Ghaffari, Mount Sinai School of Medicine, One Gustave L. Levy Place, PO Box 1496, New York, New York 10029, USA. Phone: (212) 659-8271; Fax: (212) 8036740; E-mail: saghi.ghaffari@mssm.edu. 
1. Beckman, K.B., and Ames, B.N. 1998. The free radical theory of aging matures. Physiol. Rev. 78:547-581

2. Ito, K., et al. 2004. Regulation of oxidative stress by ATM is required for self-renewal of haematopoietic stem cells. Nature. 431:997-1002.

3. Cumming, R.C., et al. 2001. Fanconi anemia group $\mathrm{C}$ protein prevents apoptosis in hematopoietic cells through redox regulation of GSTP1. Nat. Med. 7:814-820.

4. Lin, K., Dorman, J.B., Rodan, A., and Kenyon, C. 1997. daf-16: An HNF-3/forkhead family member that can function to double the life-span of Caenorhabditis elegans. Science. 278:1319-1322.

5. Lehtinen, M.K., et al. 2006. A conserved MSTFOXO signaling pathway mediates oxidativestress responses and extends life span. Cell. 125:987-1001.

6. Greer, E.L., and Brunet, A. 2005. FOXO transcription factors at the interface between longevity and tumor suppression. Oncogene. 24:7410-7425

7. Kops, G.J., et al. 2002. Forkhead transcription factor FOXO3a protects quiescent cells from oxidative stress. Nature. 419:316-321.

8. Nemoto, S., and Finkel, T. 2002. Redox regulation of forkhead proteins through a p66shc-dependent signaling pathway. Science. 295:2450-2452.

9. Dijkers, P.F., et al. 2000. Forkhead transcription factor FKHR-L1 modulates cytokine-dependent transcriptional regulation of p27(KIP1). Mol. Cell. Biol. 20:9138-9148.

10. Martinez-Gac, L., Marques, M., Garcia, Z., Campanero, M.R., and Carrera, A.C. 2004. Control of cyclin G2 mRNA expression by forkhead transcription factors: novel mechanism for cell cycle control by phosphoinositide 3-kinase and forkhead. Mol. Cell. Biol. 24:2181-2189.

11. Tran, H., et al. 2002. DNA repair pathway stimulated by the forkhead transcription factor $\mathrm{FOXO} 3$ through the Gadd45 protein. Science. 296:530-534.

12. Brunet, A., et al. 1999. Akt promotes cell survival by phosphorylating and inhibiting a Forkhead transcription factor. Cell. 96:857-868.

13. Ghaffari, S., Jagani, Z., Kitidis, C., Lodish, H.F., and Khosravi-Far, R. 2003. Cytokines and BCR-ABL mediate suppression of TRAIL-induced apoptosis through inhibition of forkhead FOXO3a transcription factor. Proc. Natl. Acad. Sci. U. S. A. 100:6523-6528.

14. You, H., and Mak, T.W. 2005. Crosstalk between p53 and FOXO transcription factors. Cell Cycle. 4:37-38.

15. Castrillon, D.H., Miao, L., Kollipara, R., Horner, J.W., and DePinho, R.A. 2003. Suppression of ovarian follicle activation in mice by the transcription factor Foxo3a. Science. 301:215-218.

16. Hosaka, T., et al. 2004. Disruption of forkhead transcription factor (FOXO) family members in mice reveals their functional diversification. Proc. Natl. Acad. Sci. U. S. A. 101:2975-2980.

17. Lin, L., Hron, J.D., and Peng, S.L. 2004. Regulation of NF-kappaB, Th activation, and autoinflammation by the forkhead transcription factor Foxo3a. Immunity. 21:203-213.

18. Kashii, Y., et al. 2000. A member of Forkhead family transcription factor, FKHRL1, is one of the downstream molecules of phosphatidylinositol 3-kinase-Akt activation pathway in erythropoietin signal transduction. Blood. 96:941-949.

19. Mahmud, D.L., et al. 2002. Phosphorylation of forkhead transcription factors by erythropoietin and stem cell factor prevents acetylation and their interaction with coactivator $\mathrm{p} 300$ in erythroid pro- genitor cells. Oncogene. 21:1556-1562.

20. Fernandez de Mattos, S., et al. 2004. FoxO3a and BCR-ABL regulate cyclin D2 transcription through a STAT5/BCL6-dependent mechanism. Mol. Cell. Biol. 24:10058-10071.

21. Constantinescu, S.N., Ghaffari, S., and Lodish, H.F. 1999. The erythropoietin receptor: structure, activation, and intracellular signal transduction. Trends Endocrinol. Metab. 10:18-23.

22. Winterbourn, C.C. 1990 . Oxidative denaturation in congenital hemolytic anemias: the unstable hemoglobins. Semin. Hematol. 27:41-50.

23. Hebbel, R.P., and Eaton, J.W. 1989. Pathobiology of heme interaction with the erythrocyte membrane. Semin. Hematol. 26:136-149.

24. Friedman, J.S., et al. 2001. Absence of mitochondrial superoxide dismutase results in a murine hemolytic anemia responsive to therapy with a catalytic antioxidant. J. Exp. Med. 193:925-934.

25. Lee, J.M., Chan, K., Kan, Y.W., and Johnson, J.A 2004. Targeted disruption of Nrf2 causes regenerative immune-mediated hemolytic anemia. Proc Natl. Acad. Sci. U. S. A. 101:9751-9756.

26. Johnson, R.M., Goyette, G., Jr. Ravindranath, Y., and Ho, Y.S. 2005. Hemoglobin autoxidation and regulation of endogenous $\mathrm{H} 2 \mathrm{O} 2$ levels in erythrocytes. Free Radic. Biol. Med. 39:1407-1417.

27. Steinberg, M.H., et al. 2000. Pathobiology of the human erythrocyte and its hemoglobins. In Hematology: basic principles and practice. E.J. Benz et al., editors. 3rd edition. Churchill Livingstone. Oxford, United Kingdom. 356-367.

28. Zhao, W., Kitidis, C., Fleming, M.D., Lodish, H.F., and Ghaffari, S. 2006. Erythropoietin stimulates phosphorylation and activation of GATA-1 via the PI3-kinase/AKT signaling pathway. Blood. 107:907-915

29. Ghaffari, S., et al. 2006. AKT induces erythroid-cell maturation of JAK2-deficient fetal liver progenitor cells and is required for Epo regulation of erythroid-cell differentiation. Blood. 107:1888-1891.

30. Itano, H.A., Hirota, K., and Hosokawa, K. 1975. Mechanism of induction of haemolytic anaemia by phenylhydrazine. Nature. 256:665-667.

31. Nakamura, A., and Goto, S. 1996. Analysis of protein carbonyls with 2,4-dinitrophenyl hydrazine and its antibodies by immunoblot in two-dimensional gel electrophoresis. J. Biochem. (Tokyo). 119:768-774

32. Hoffmann-Fezer, G., et al. 1993. Biotin labeling as an alternative nonradioactive approach to determination of red cell survival. Ann. Hematol. 67:81-87.

33. Qanungo, S., Wang, M., and Nieminen, A.L. 2004. $\mathrm{N}$-acetyl-L-cysteine enhances apoptosis through inhibition of nuclear factor-kappaB in hypoxic murine embryonic fibroblasts. J. Biol. Chem. 279:50455-50464.

34. Socolovsky, M., et al. 2001. Ineffective erythropoiesis in Stat5a(-/-) $5 \mathrm{~b}(-/-)$ mice due to decreased survival of early erythroblasts. Blood. 98:3261-3273.

35. Welch, J.J., et al. 2004. Global regulation of erythroid gene expression by transcription factor GATA-1. Blood. 104:3136-3147.

36. Hsieh, F.F., et al. 2000. Cell cycle exit during terminal erythroid differentiation is associated with accumulation of p27(Kip1) and inactivation of cdk2 kinase. Blood. 96:2746-2754.

37. Fraser, J.K., Nicholls, J., Coffey, C., Lin, F.K., and Berridge, M.V. 1988. Down-modulation of highaffinity receptors for erythropoietin on murine erythroblasts by interleukin 3. Exp. Hematol. 16:769-773.

38. Zhang, J., Socolovsky, M., Gross, A.W., and Lodish,
H.F. 2003. Role of Ras signaling in erythroid differentiation of mouse fetal liver cells: functional analysis by a flow cytometry-based novel culture system. Blood. 102:3938-3946.

39. Ghaffari, S., et al. 2001. Erythropoiesis in the absence of janus-kinase 2: BCR-ABL induces red cell formation in JAK2 $(-/-)$ hematopoietic progenitors. Blood. 98:2948-2957.

40. Alvarez, B., Garrido, E., Garcia-Sanz, J.A., and Carrera, A.C. 2003. Phosphoinositide 3-kinase activation regulates cell division time by coordinated control of cell mass and cell cycle progression rate. J. Biol. Chem. 278:26466-26473.

41. Seoane, J., Le, H.V., Shen, L., Anderson, S.A., and Massague, J. 2004. Integration of Smad and forkhead pathways in the control of neuroepithelial and glioblastoma cell proliferation. Cell. 117:211-223.

42. O'Reilly, M.A. 2005. Redox activation of p21Cip1/ WAF1/Sdi1: a multifunctional regulator of cell survival and death. Antioxid. Redox Signal. 7:108-118.

43. Sablina, A.A., et al. 2005. The antioxidant function of the p53 tumor suppressor. Nat. Med. 11:1306-1313.

44. Fang, L., et al. 1999. p21Waf1/Cip1/Sdi1 induces permanent growth arrest with markers of replicative senescence in human tumor cells lacking functional p53. Oncogene. 18:2789-2797.

45. Budanov, A.V., Sablina, A.A., Feinstein, E., Koonin, E.V., and Chumakov, P.M. 2004. Regeneration of peroxiredoxins by $\mathrm{p} 53$-regulated sestrins, homologs of bacterial AhpD. Science. 304:596-600.

46. Bauer, M., and Bauer, I. 2002. Heme oxygenase-1: redox regulation and role in the hepatic response to oxidative stress. Antioxid. Redox Signal. 4:749-758.

47. Macip, S., et al. 2002. Inhibition of p21-mediated ROS accumulation can rescue $\mathrm{p} 21$-induced senescence. EMBO J. 21:2180-2188

48. Hara, H., and Ogawa, M. 1976. Erthropoietic precursors in mice with phenylhydrazine-induced anemia. Am. J. Hematol. 1:453-458.

49. Furuyama, T., Nakazawa, T., Nakano, I., and Mori, N. 2000. Identification of the differential distribution patterns of mRNAs and consensus binding sequences for mouse DAF-16 homologues. Biochem. J. 349:629-634.

50. Gregory, C.J., and Eaves, A.C. 1978. Three stages of erythropoietic progenitor cell differentiation distinguished by a number of physical and biologic properties. Blood. 51:527-537.

51. Iscove, N.N. 1977. The role of erythropoietin in regulation of population size and cell cycling of early and late erythroid precursors in mouse bone marrow. Cell Tissue Kinet. 10:323-334.

52. Goardon, N., et al. 2006. ETO2 coordinates cellular proliferation and differentiation during erythropoiesis. EMBOJ. 25:357-366

53. Bakker, W.J., et al. 2004. FoxO3a regulates erythroid differentiation and induces BTG1, an activator of protein arginine methyl transferase 1. J. Cell Biol. 164:175-184

54. Barzilai, A., Rotman, G., and Shiloh, Y. 2002. ATM deficiency and oxidative stress: a new dimension of defective response to DNA damage. DNA Repair (Amst.). 1:3-25.

55. Tothova, Z., et al. 2007. FoxOs are critical mediators of hematopoietic stem cell resistance to physiologic oxidative stress. Cell. 128:325-339.

56. Chen, X., and Bieker,J.J. 2004. Stage-specific repression by the EKLF transcriptional activator. $\mathrm{Mol}$. Cell. Biol. 24:10416-10424.

57. Manders, E.M.M., Verbeek, F.J., and Aten, J.A. 1993. Measurement of co-localization of objects in dualcolor confocal images. J. Microsc. 169:375-382. 